\title{
Malignant Myoepithelioma of Vulva; How Rare is it? : Case Report
}

\author{
Dr. Fatema Alkhan ${ }^{1}$, Dr. Emine Karabuk ${ }^{1}$, Dr. M. Murat Naki ${ }^{1}$, Dr. Mete Gungor ${ }^{2}$, \\ Dr. M. Faruk Kose ${ }^{1}$
}

\section{Acibadem University Atakent Hospital ${ }^{1}$ Acibadem Maslak Hospital ${ }^{2}$}

Introduction:

Malignant myoepethelial cells are rare cancer cells that commonly occur within the salivary glands. It rarely occurs in the vulva (1). These tumors are composed of epitheliod, plasmatoctoid, spindle-shaped, and clear cells with a characteristic of microscope and immunohistochemical appearance (2). The PubMed database was chosen for research, as it is the most widely used resource for medical literature. The following terms were used for myoepithelioma of the vulva and nine literatures were found.

Case:

A 64-year-old woman came to our center for a second opinion after a biopsy result. She was complaining of a non-painful swelling of the left labia majora for six months. After that, she had excisional biopsy. The pathology results revealed Epitheliod Sarcoma. Therefore, the case was discussed in the Tumor Board Meeting and the decision was made for radical vulvectomy with bilateral inguinofemoral lymphadenectomy. We performed the needed surgery on the patient. The pathologist requested the original pathology specimens. The re-review of the original pathology slides showed the myoepithelioma of the vulva. The case was again discussed in the Tumor Board Meeting and recommended for an adjuvant radiotherapy.

Discussion:

Malignant myoepithelioma of the vulva are rare tumors and need an expert pathologist. The Pathologist requested to review the first biopsy result that reported Epitheliod sarcoma and our surgery pathology results revealed myoepthelioma. Therefore, all available glass slides and pathology reports were re-reviewed. Immunohistochemically, the tumor cells were positive for these staining EMA, Actin (SMA), Pan-ck (5, 6, 8, 18), CD 34 and Calponin; whereas it was negative for GFAP and TLE 1.
There were four mitosis figures per ten high-power field (HPF) and the Ki-69 index was 25\%. Therefore, based on that, the final diagnosis was malignant myoepithelioma. The myoepithelioma can present as low or high-grade malignancy, and the grade is the one that determines the overall prognosis. Optimal treatment should comprise radical excision of the primary site, bilateral inguinal lymph node dissection and adjuvant radiotherapy (3).

Conclusion:

An Expert pathologist is needed in this field due to the rarity of myoepitheloma. It would be more helpful if there was a bank for soft tissue sarcoma registration. So, sharing the experiences and reviewing the cases may help for better management and guidelines.

\section{References:}

1. Meenakshi M, McCluggage W. G. Myoepithelial neoplasms involving the vulva and vagina: report of 4 cases. Human Pathology 2009; 17471753

2. Kyriazi M. A, Carvounis E. E, Kitsou M, Arkadopoulos N, Nicolaidou E, Fotiou S, Smyrniotis V. Myoepithelial carcinoma of the vulva mimicking Bartholin gland abscess in a pregnant woman: case report and review of literature. International Journal of Gynecological Pathology 2010; 29-501-504

3. Fukunaga M. Myoepithelioma of the vulva. APMIS 2003; 111: 41620 